\title{
Quasistatic viscoelasticity with self-contact at large strains
}

\author{
Stefan Krömer!1 and Tomáš Roubíček ${ }^{2}: 3$
}

\begin{abstract}
The frame-indifferent viscoelasticity in Kelvin-Voigt rheology at large strains is formulated in the reference configuration (i.e. using the Lagrangian approach) considering also the possible self-contact in the actual deformed configuration. Using the concept of 2nd-grade nonsimple materials, existence of certain weak solutions which are a.e. injective is shown by converging an approximate solution obtained by the implicit time discretisation.
\end{abstract}

Keywords: Kelvin-Voigt material, frame indifference, non-selfinterpenetration, implicit time discretisation, Lagrangian description, pullback.

AMS Clasification: 35K86, 35Q74, 74A30, 74B20, 74M15.

\section{Introduction}

Nonlinear elasticity and viscoelasticity is a vital part of the continuum mechanics of solids and still faces many open fundamental problems even after intensive scrutiny within past many decades. One of such problem is the possibility of non-physical self-interpenetration and analytically supported methods to prevent it. The problem is difficult because of an interaction of two configurations: the reference one (ultimately needed for analysis of problems in solid mechanics at large strains) and the actual one (ultimately need for determination the possible time-varying self-contact boundary region).

So far, besides merely static situations, only rate-independent evolution of some internal variables based on (not always very realistic) concept of instantaneous global minimization and energetic solutions, possessing a good variational structure and thus allowing incorporation of the Ciarlet-Nečas condition [4], has been treated in [10]. In the viscoelasticity, one cannot rely purely on a variational structure but should rather work in terms of partial differential equations. As emphasized in [8, 9], "the theory of viscoelasticity at finite strain is notoriously difficult and" that time it seemed "that the present mathematical tools are not sufficient to provide sufficiently strong solutions in the multidimensional, truly geometrically invariant case". Since then, the quasistatic viscoelasticity has been treated in [11] and in the dynamical variant in [7, Sect.9.3], but without globally ruling out self-interpenetration.

In the case of self-contact, instead of differential equations, it is natural to describe static critical points by variational inequalities. This was developed for a purely static situation in [14] for non-simple materials involving a higher order term in the energy. For the case of a static obstacle problem neglecting possible self-contact and self-interpenetration see also [18].

The goal of this article is to merge the results of A.Z. Palmer and T.J. Healey [14] with the evolution viscoelastic model from [11, using a generalization of Korn's inequality developed by

\footnotetext{
${ }^{1}$ Institute of Information Theory and Automation, Czech Acad. Sci., Pod vodárenskou věží 4, CZ-182 08 Praha 8, Czech Republic

${ }^{2}$ Charles University, Mathematical Institute, Sokolovská 83, CZ-186 75 Praha 8, Czech Republic

${ }^{3}$ Institute of Thermomechanics, Czech Acad. Sci., Dolejškova 5, CZ-182 08 Praha 8, Czech Republic
} 
P. Neff and W. Pompe [13, 16]. By this, we obtain first results for viscoelastic model allowing a self-contact while respecting non-self-interpenetration. Let us point out that for a long time, this was an open problem and largely ignored in engineering numerical calculations which admitted interpenetration, relying solely on the fact that for particular scenarios, computational simulations are often not likely to go into such non-physical situations.

The plan of the article is following: In Sect.2, we formulate the problem in terms of the classical partial differential equations, together with its weak form. Then, in Sect.3, we employ a time discretisation, prove basic a-priori estimates and, by convergence for time-step approaching zero, prove existence of a weak solution. At this point, local non-selfinterpenetration and avoidance of singularities while keeping the deformation gradient "uniformly" invertible everywhere is granted by using the so-called 2nd-grade non-simple (i.e. involving strain-gradient) material concept and the results from [6].

\section{Quasistatic viscoelasticity}

Strain-gradient theories describe materials referred to as nonsimple, or also multipolar or complex. This concept has been introduced a long time ago, cf. [20,21] or also e.g. [3, 5, 15, 19, 22, .

We will use the Lagrangian approach and formulate the model in the reference (fixed) domain $\Omega \subset \mathbb{R}^{d}$ with a smooth boundary $\Gamma:=\partial \Omega, d \geq 2$.

To introduce our model in a broader context, we may define the total free energy and the total dissipation potential

$$
\Psi(y)= \begin{cases}\int_{\Omega} \varphi(\nabla y)+\mathscr{H}\left(\nabla^{2} y\right) \mathrm{d} x & \text { if } \int_{\Omega} \operatorname{det} \nabla y \mathrm{~d} x \leq \text { meas } y(\Omega), \\ +\infty & \text { otherwise }\end{cases}
$$

and

$$
\mathcal{R}(y, \dot{y})=\int_{\Omega} \zeta(\nabla y, \nabla \dot{y}) \mathrm{d} x
$$

respectively. The condition $\int_{\Omega} \operatorname{det} \nabla y \mathrm{~d} x \leq$ meas $y(\Omega)$ involved in (2.1a) is called the CiarletNečas condition [4]. Together with $\operatorname{det} \nabla y \geq 0$ on $\Omega$ as a property which can (and will) be ensured by the strain energy $\varphi$, it guarantees global non-interpenetration.

The mechanical evolution part can then be viewed as an abstract gradient flow

$$
\partial_{\dot{y}} \mathcal{R}(y, \dot{y})+\Psi^{\prime}(y)=\mathcal{F}(t) \text { with }\langle\mathcal{F}, y\rangle=\int_{\Omega} f(x, t) \cdot y(x) \mathrm{d} x,
$$

cf. also [8,23] for the case without the Ciarlet-Nečas condition. The sum of the conservative and the dissipative parts corresponds to the Kelvin-Voigt rheological model in the quasistatic variant (neglecting inertia). Here and henceforth, the notation " $\partial$ " is used for partial derivatives (here functional, or later in Euclidean spaces), while $(\cdot)^{\prime}$ is used for the derivative of functions of only one variable.

The generalized gradient $\Psi^{\prime}$ is to be understood rather formally due to the integral CiarletNečas constraint. This constraint gives rise to the reaction force due to a possible self-contact. The contact zone is time evolving and not a-priori known, which is in some sense a generalization of a so-called Hertz contact at small strains. At large strains, one must distinguish between the actual deforming configuration which is relevant for the contact and the reference configuration which is to be used for analysis and for formulation of the boundary conditions. Here we use the 
results of Palmer and Healey that describe the boundary forces that arise due to the constraint in a static situation [14.

Writing (2.2) locally in the classical formulation, one arrives at the nonlinear parabolic 4thorder partial differential equation expressing quasistatic momentum equilibrium,

$$
\operatorname{div} \sigma+g=0 \quad \text { with } \quad \sigma=\sigma_{\mathrm{vi}}+\sigma_{\mathrm{el}}-\operatorname{div} \mathfrak{h}_{\mathrm{el}},
$$

where the viscous stress is $\sigma_{\mathrm{vi}}=\sigma_{\mathrm{vi}}(F, \dot{F})$ and the elastic stress is $\sigma_{\mathrm{el}}=\sigma_{\mathrm{el}}(F)$ with $F$ a placeholder for the deformation gradient $\nabla y$ and $\dot{F}$ a placeholder for its time derivative, while $\mathfrak{h}_{\mathrm{el}}$ is a so-called hyperstress arising from the 2nd-grade nonsimple-material concept, cf. e.g. $[15,19,20]$. In view of the local potentials used in (2.2), we have

$$
\sigma_{\mathrm{vi}}(F, \dot{F})=\partial_{\dot{F}} \zeta(F, \dot{F}), \quad \sigma_{\mathrm{el}}(F)=\varphi^{\prime}(F), \quad \text { and } \quad \mathfrak{h}_{\mathrm{el}}(\mathrm{G})=\mathscr{H}^{\prime}(\mathrm{G}),
$$

where $\mathrm{G} \in \mathbb{R}^{d \times d \times d}$ is a placeholder for $\nabla F$, i.e. for $\nabla^{2} y$.

An important physical requirement is static and dynamic frame indifference. For the elastic stresses, static frame indifference means that

$$
\sigma_{\mathrm{el}}(R F)=R \sigma_{\mathrm{el}}(F) \quad \text { and } \quad \mathfrak{h}_{\mathrm{el}}(R \mathrm{G})=R \mathfrak{h}_{\mathrm{el}}(\mathrm{G})
$$

for all $R \in \mathrm{SO}(d), F$ and $\mathrm{G}$. For the viscous stresses, dynamic frame indifference means that

$$
\sigma_{\mathrm{vi}}(R F, \dot{R} F+R \dot{F})=R \sigma_{\mathrm{vi}}(F, \dot{F})
$$

for all smoothly time-varying $R: t \mapsto R(t) \in \mathrm{SO}(d)$ and $F: t \mapsto F(t) \in G L^{+}(d)$, cf. [1]. Note that $R$ may depend on $t$ but not on $x \in \Omega$, since frame-indifference relates to superimposing time-dependent rigid-body motions.

In terms of the thermodynamic potentials $\zeta, \varphi$, and $\mathscr{H}$, these frame indifferences read as

$$
\begin{aligned}
& \varphi(R F)=\varphi(F), \quad \mathscr{H}(R G)=\mathscr{H}(G), \quad \text { and } \\
& \zeta\left(R F ;(R F)^{\circ}\right)=\zeta(R F ; \dot{R} F+R \dot{F})=\zeta(F ; \dot{F})
\end{aligned}
$$

for $R, F$ and $G$ as above.

As to $\zeta$, the simplest possible choice with such a frame indifference leads to a viscosity $\sigma_{\mathrm{vi}}=\partial_{\dot{F}} \zeta$ which is linear in $\dot{F}$, while its associated potential is quadratic:

$$
\zeta(F ; \dot{F}):=\frac{1}{2} \hat{\mathbb{D}}(C) \dot{C}: \dot{C} \quad \text { where } \quad C:=F^{\top} F \quad \text { and } \quad \dot{C}:=\dot{F}^{\top} F+F^{\top} \dot{F} .
$$

Notice that frame indifference in (2.7) is built in by using a potential which only depends on the right Cauchy-Green tensor $C$ and its formal time derivative $\dot{C}$. To avoid unnecessary technicalities, we adopt this kind of viscosity term for the rest of the paper. Although the material viscosity is linear as a consequence of (2.7), the geometrical nonlinearity arising from large strains is still a vital part of the problem since $\sigma_{\mathrm{vi}}(F, \dot{F})$ depends on $F$, too.

Altogether, denoting $\mathbb{D}(F):=\hat{\mathbb{D}}\left(F^{\top} F\right)$, we arrive at the parabolic problem

$$
\begin{gathered}
\operatorname{div}\left(\sigma_{\mathrm{vi}}(\nabla y, \nabla \dot{y})+\sigma_{\mathrm{el}}(\nabla y)-\operatorname{div} \mathscr{H}^{\prime}\left(\nabla^{2} y\right)\right)+f=0 \\
\text { with } \sigma_{\mathrm{vi}}(F, \dot{F}):=2 F \mathbb{D}(F)\left(F^{\top} \dot{F}+\dot{F}^{\top} F\right) \\
\text { and } \sigma_{\mathrm{el}}(F)=\varphi^{\prime}(F),
\end{gathered}
$$


on $Q$.

We complete (2.8) by some boundary conditions. For simplicity, we only consider a mechanically fixed part $\Gamma_{\mathrm{D}}$, undeformed and independent of time (i.e. identity):

$$
\begin{array}{ll}
y(x)=x \quad \text { (identity) } & \text { on } \Gamma_{\mathrm{D}}, \\
\left(\sigma_{\mathrm{vi}}(\nabla y, \nabla \dot{y})+\sigma_{\mathrm{el}}(\nabla y)\right) \vec{n}-\operatorname{div}_{\mathrm{S}}\left(\mathscr{H}^{\prime}\left(\nabla^{2} y\right) \vec{n}\right)=\mathfrak{s} \quad(\text { a reaction force) } & \text { on } \Gamma_{\mathrm{N}}, \\
\mathscr{H}^{\prime}\left(\nabla^{2} y\right):(\vec{n} \otimes \vec{n})=0 & \text { on } \Gamma,
\end{array}
$$

where $\Gamma=\partial \Omega, \Gamma_{\mathrm{N}}=\Gamma \backslash \Gamma_{\mathrm{D}}$ and $\vec{n}$ is the outward pointing normal vector. Moreover, "div ${ }_{\mathrm{S}}$ " in (2.9b) denotes the surface divergence defined as $\operatorname{div}_{\mathrm{S}}(\cdot)=\operatorname{tr}\left(\nabla_{\mathrm{S}}(\cdot)\right)$, where $\operatorname{tr}(\cdot)$ denotes the trace and $\nabla_{\mathrm{S}}$ denotes the surface gradient given by $\nabla_{\mathrm{S}} v=(\mathbb{I}-\vec{n} \otimes \vec{n}) \nabla v=\nabla v-\frac{\partial v}{\partial \vec{n}} \vec{n}$. Note that for equilibria, (2.9b) and (2.9c) reduce to the natural boundary conditions complementing the Dirichlet condition (2.9a).

The energetics of the system (2.8)-(2.9) can be revealed by testing (2.8) by $\dot{y}$, and using the boundary conditions after integration over $\Omega$ and using Green's formula twice together with another $(d-1)$-dimensional Green formula over $\Gamma$ for (2.8). The last mentioned technique is related with the concept of nonsimple materials; for the details about how the boundary conditions are handled see e.g. [17, Sect. 2.4.4]. This test of (2.8) gives the mechanical energy balance:

$$
\int_{\Omega} \underbrace{2 \zeta(\nabla y, \nabla \dot{y})}_{\begin{array}{c}
\text { dissipation } \\
\text { rate }
\end{array}}+\underbrace{\sigma_{\text {el }}(\nabla y): \nabla \dot{y}}_{\begin{array}{c}
\text { mechanical } \\
\text { power }
\end{array}} \mathrm{d} x+\frac{\mathrm{d}}{\mathrm{d} t} \int_{\begin{array}{c}
\Omega \\
\text { "nonsimple" part of } \\
\text { the stored energy }
\end{array}}^{\mathscr{H}\left(\nabla^{2} y\right)} \mathrm{d} x=\int_{\begin{array}{c}
\Omega \\
\text { power of the } \\
\text { bulk force }
\end{array}}^{f \cdot \dot{y}} \mathrm{~d} x .
$$

In what follows, we will use the (standard) notation for the Lebesgue $L^{p}$-spaces and $W^{k, p}$ for Sobolev spaces whose $k$-th distributional derivatives are in $L^{p}$-spaces and the abbreviation $H^{k}=W^{k, 2}$. The notation $W_{\mathrm{D}}^{1, p}$ will indicate the closed subspace of $W^{1, p}$ with zero traces on $\Gamma_{\mathrm{D}}$. The Banach space of continuous functions on a compact set will be denoted as $C(\cdot)$, while their dual as Meas $(\cdot)$ being the space of finite Radon measures; if scalar-valued, the subset of nonnegative measures will be denoted by $\mathrm{Meas}^{+}(\cdot)$. Moreover, we will use the standard notation $p^{\prime}=p /(p-1)$. In the vectorial case, we will write $L^{p}\left(\Omega ; \mathbb{R}^{n}\right) \cong L^{p}(\Omega)^{n}$ and $W^{1, p}\left(\Omega ; \mathbb{R}^{n}\right) \cong$ $W^{1, p}(\Omega)^{n}$. For the fixed time interval $I=[0, T]$, we denote by $L^{p}(I ; X)$ the standard Bochner space of Bochner-measurable mappings $I \rightarrow X$ with $X$ a Banach space whose norm is in $L^{p}(I)$. Also, $W^{k, p}(I ; X)$ denotes the Banach space of mappings from $L^{p}(I ; X)$ whose $k$-th distributional derivative in time is also in $L^{p}(I ; X)$. The dual space to $X$ will be denoted by $X^{*}$. Moreover, $C_{\mathrm{w}}(I ; X)$ denotes the Banach space of weakly continuous functions $I \rightarrow X$, and $L_{\mathrm{w}}^{\infty}(I ; X)$ denotes the Banach space of essentially bounded, weakly measurable functions $I \rightarrow X$. The scalar product between vectors, matrices, or 3rd-order tensors will be denoted by ".", ":", or " : ", respectively. Finally, in what follows, $K$ denotes a positive, possibly large constant.

We consider an initial-value problem, imposing the initial conditions

$$
y(0, \cdot)=y_{0} \quad \text { on } \Omega .
$$

Definition 2.1 (Weak solution) The couple $(y, \mathfrak{s})$ of a displacement field $y: Q \rightarrow \mathbb{R}^{d}$ and a reaction traction $\mathfrak{s}$ as a distribution $\Sigma_{\mathrm{N}} \rightarrow \mathbb{R}^{d}$ is called a weak solution of the constrained initial-boundary-value problem (2.8)-(2.9)-(2.11) if the following three conditions are satisfied: 
(i) $(y, \mathfrak{s}) \in C_{\mathrm{w}}\left(I ; W^{2, p}\left(\Omega ; \mathbb{R}^{d}\right)\right) \times L^{2}\left(I ; W^{2-1 / p, p}\left(\Gamma_{\mathrm{N}} ; \mathbb{R}^{d}\right)^{*}\right)$ with $\nabla \dot{y} \in L^{2}\left(Q ; \mathbb{R}^{d \times d}\right)$ and with $\min _{Q} \operatorname{det} \nabla y>0$ and $\left.y\right|_{\Sigma_{\mathrm{D}}}=$ identity, and the integral identity

$$
\begin{aligned}
\int_{Q} \mathbb{D}(\nabla y)\left(\nabla \dot{y}^{\top} \nabla y+\nabla y^{\top} \nabla \dot{y}\right) & \left(\nabla y^{\top} \nabla z+\nabla z^{\top} \nabla y\right)+\varphi^{\prime}(\nabla y): \nabla z \\
& +\mathscr{H}^{\prime}\left(\nabla^{2} y\right) \vdots \nabla^{2} z \mathrm{~d} x \mathrm{~d} t=\int_{Q} f \cdot z \mathrm{~d} x \mathrm{~d} t+\left\langle\mathfrak{s},\left.z\right|_{\Sigma_{\mathrm{N}}}\right\rangle
\end{aligned}
$$

is satisfied for all smooth $z: Q \rightarrow \mathbb{R}^{d}$ with $z=0$ on $\Sigma_{\mathrm{D}}$ together with $y(0, \cdot)=y_{0}$.

(ii) For a.e. $t \in I, y(t, \cdot)$ satisfies the Ciarlet-Nečas condition, i.e.,

$$
\int_{\Omega} \operatorname{det} \nabla y(t, x) \mathrm{d} x \leq \text { meas } y(t, \Omega)
$$

(iii) The support of $\mathfrak{s}$ is contained in the part of $\Sigma_{\mathrm{N}}$ which, after deformation, is in self-contact:

$$
\langle\mathfrak{s}(t, \cdot), z\rangle=0 \quad \text { for a.e. } t \in I \text { and every } z \in W^{2-1 / p, p}\left(\Gamma_{\mathrm{N}} ; \mathbb{R}^{d}\right) \text { vanishing on } \mathfrak{S}_{t},
$$

where the self-contact set at time $t$ given by

$$
\mathfrak{S}_{t}:=\left\{x \in \Gamma_{\mathrm{N}} \mid \exists \tilde{x} \in \bar{\Omega} \backslash\{x\}: y(t, x)=y(t, \tilde{x})\right\} .
$$

Remark 2.2 (The role of $\mathfrak{s}$ ) The constraint (2.12b), effectively ruling out self-interpenetration, is also built into our definition (2.1a) of the total free energy $\Psi$. The reaction force $\mathfrak{s}$ has the role of a Lagrange multiplier which only appears at the "boundary" of this constraint, i.e., when there is self-contact.

Remark 2.3 (Frame indifference and more general viscosity terms) Even in the case when $\zeta$ is not quadratic, the frame indifferences (2.6) imply (cf. [2,8]) the existence of reduced potentials $\hat{\varphi}, \hat{\zeta}$, and $\hat{\mathscr{H}}$ such that

$$
\zeta(F, \dot{F})=\hat{\zeta}(C, \dot{C}), \quad \varphi(F)=\hat{\varphi}(C), \quad \text { and } \quad \mathscr{H}(\mathrm{G})=\hat{\mathscr{H}}(\mathrm{B})
$$

where $\mathrm{B}=\mathrm{G}^{\top} \cdot \mathrm{G} \in \mathbb{R}^{(d \times d) \times(d \times d)}$, and $C \in \mathbb{R}_{\mathrm{sym}}^{d \times d}$ is the right Cauchy-Green tensor with time derivative $\dot{C}$ as in (2.7). More specifically, denoting $\mathrm{G}=\left[\mathrm{G}_{\alpha i j}\right]$ the placeholder for $\frac{\partial}{\partial x_{j}} F_{\alpha i}$ with $F_{\alpha i}$ the placeholder for $\frac{\partial}{\partial x_{i}} y_{\alpha}$, the exact meaning is $\left[\mathrm{G}^{\top} \cdot \mathrm{G}\right]_{i j k l}:=\sum_{\alpha=1}^{d} \mathrm{G}_{\alpha i j} \mathrm{G}_{\alpha k l}$ and $\left[F^{\top} F\right]_{i j}:=\sum_{\alpha=1}^{d} F_{\alpha i} F_{\alpha j}$. The ansatz (2.13) also means that

$$
\begin{aligned}
& \sigma_{\mathrm{el}}=2 F \partial_{C} \hat{\varphi}\left(F^{\top} F\right), \quad \mathfrak{h}_{\mathrm{el}}(\mathrm{G})=2 \mathrm{G} \partial_{\mathrm{B}} \hat{\mathscr{H}}\left(\mathrm{G}^{\top} \cdot \mathrm{G}\right)=2 \mathrm{G} \partial_{\mathrm{B}} \hat{\mathscr{H}}(\mathrm{B}), \\
& \sigma_{\mathrm{vi}}(F, \dot{F})=2 F \partial_{\dot{C}} \hat{\zeta}\left(F^{\top} F, \dot{F}^{\top} F+F^{\top} \dot{F}\right)=2 F \partial_{\dot{C}} \hat{\zeta}(C, \dot{C}) .
\end{aligned}
$$

Furthermore, the specific dissipation rate can easily be identified in terms of $\hat{\zeta}$ as

$$
\begin{aligned}
\xi(F, \dot{F}) & =\sigma_{\mathrm{vi}}(F, \dot{F}): \dot{F}=2 F \partial_{\dot{C}} \hat{\zeta}\left(F^{\top} F, \dot{F}^{\top} F+F^{\top} \dot{F}\right): \dot{F} \\
& =\partial_{\dot{C}} \hat{\zeta}\left(F^{\top} F, \dot{F}^{\top} F+F^{\top} \dot{F}\right):\left(\dot{F}^{\top} F+F^{\top} \dot{F}\right) .
\end{aligned}
$$

For our choice (2.7), we thus have $\xi(F, \dot{F})=\hat{\mathbb{D}}(C) \dot{C}: \dot{C}=2 \zeta(F ; \dot{F})$. 


\section{Analysis by time discretisation}

Let us summarize the assumptions we impose on the data:

$$
\begin{aligned}
& \exists p>d, s>1, q \geq p d /(p-d) \exists \alpha, K, \epsilon>0: \\
& \varphi: \mathrm{GL}^{+}(d) \rightarrow \mathbb{R}^{+} \text {continuously differentiable, } \forall F \in \mathrm{GL}^{+}(d): \\
& \quad \varphi(F) \geq \epsilon|F|^{s}+\epsilon /(\operatorname{det} F)^{q}, \\
& \mathscr{H}: \mathbb{R}^{d \times d \times d} \rightarrow \mathbb{R}^{+} \text {convex, continuously differentiable, } \forall G, G_{1}, G_{2} \in \mathbb{R}^{d \times d \times d}: \\
& \quad \epsilon|G|^{p} \leq \mathscr{H}(G) \leq K\left(1+|G|^{p}\right),\left|\mathscr{H}^{\prime}(G)\right| \leq K\left(1+|G|^{p-1}\right), \\
& \quad \alpha\left|G_{1}-G_{2}\right|^{p} \leq\left[\mathscr{H}^{\prime}\left(G_{1}\right)-\mathscr{H}^{\prime}\left(G_{2}\right)\right] \vdots\left(G_{1}-G_{2}\right), \\
& \zeta(F, \dot{F})=\frac{1}{2} \mathbb{D}(F)\left(F^{\top} \dot{F}+\dot{F}^{\top} F\right):\left(F^{\top} \dot{F}+\dot{F}^{\top} F\right) \text { with } \mathbb{D}(F)=\hat{\mathbb{D}}\left(F^{\top} F\right)(\mathrm{cf} \text {. (2.7) }), \\
& C \mapsto \hat{\mathbb{D}}(C) \operatorname{continuous} \text { and bounded, } \forall C, \dot{C} \in \mathbb{R}_{\mathrm{sym}}^{d \times d}: \\
& \quad \hat{\mathbb{D}}(C): \mathbb{R}_{\mathrm{sym}}^{d \times d} \rightarrow \mathbb{R}_{\mathrm{sym}}^{d \times d} \text { is linear and symmetric, } \\
& \quad \hat{\mathbb{D}}(C) \dot{C}: \dot{C} \geq \alpha|\dot{C}|^{2}, \\
& f \in H^{1}\left(I ; L^{2}\left(\Omega ; \mathbb{R}^{d}\right)\right), \\
& y_{0} \in W^{2, p}\left(\Omega ; \mathbb{R}^{d}\right), \quad \operatorname{det}\left(\nabla y_{0}\right) \geq \epsilon,\left.\quad y_{0}\right|_{\Gamma_{\mathrm{D}}}=\text { identity, }
\end{aligned}
$$

where $\mathrm{GL}^{+}(d)$ denotes the set of matrices in $\mathbb{R}^{d \times d}$ with positive determinant.

The balance of the mechanical energy (2.10) can be written in the more specific form

$$
\int_{\Omega} \underbrace{\zeta(\nabla y, \nabla \dot{y})}_{\begin{array}{c}
\text { dissipation } \\
\text { rate }
\end{array}} \mathrm{d} x+\frac{\mathrm{d}}{\mathrm{d} t} \int_{\Omega} \underbrace{\varphi(\nabla y)+\mathscr{H}\left(\nabla^{2} y\right)}_{\begin{array}{c}
\text { stored } \\
\text { energy }
\end{array}} \mathrm{d} x=\int_{\Omega} \underbrace{f \cdot \dot{y}}_{\begin{array}{c}
\text { power of } \\
\text { bulk load }
\end{array}} \mathrm{d} x .
$$

Our main result is the following:

Theorem 3.1 (Existence of weak solutions) Let (3.1) hold. Then there exists a weak solution to the constrained initial-boundary-value problem (2.8)-(2.9)-(2.11) in the sense of Definition 2.1.

Proof. As we have neglected inertial effects, we can use time discretisation. We consider a time step $\tau>0$ such that $T / \tau$ is integer, and an equidistant partition of the time interval $I=[0, T]$. Thus the regularized system (2.8) $-(2.9)$ after this discretisation takes the form

$$
\begin{aligned}
& -\operatorname{div}\left(\sigma_{\mathrm{vi}}\left(\nabla y_{\tau}^{k-1}, \nabla \frac{y_{\tau}^{k}-y_{\tau}^{k-1}}{\tau}\right)+\sigma_{\mathrm{el}}\left(\nabla y_{\tau}^{k}\right)\right. \\
& \left.-\operatorname{div} \mathscr{H}^{\prime}\left(\nabla^{2} y_{\tau}^{k}\right)\right)=f_{\tau}^{k}:=\frac{1}{\tau} \int_{(k-1) \tau}^{k \tau} f(t) \mathrm{d} t \quad \text { on } \Omega, \\
& \left(\sigma_{\mathrm{vi}}\left(\nabla y_{\tau}^{k-1}, \nabla \frac{y_{\tau}^{k}-y_{\tau}^{k-1}}{\tau}\right)+\sigma_{\mathrm{el}}\left(\nabla y_{\tau}^{k}\right)\right) \vec{n}-\operatorname{div}_{\mathrm{S}}\left(\mathscr{H}^{\prime}\left(\nabla^{2} y_{\tau}^{k}\right) \vec{n}\right)=\mathfrak{s}_{\tau}^{k} \quad \text { on } \Gamma_{\mathrm{N}} \text {, } \\
& y_{\tau}^{k}=\text { identity on } \Gamma_{\mathrm{D}}, \quad \quad \mathscr{H}^{\prime}\left(\nabla^{2} y_{\tau}^{k}\right):(\vec{n} \otimes \vec{n})=0 \quad \text { on } \Gamma \text {, }
\end{aligned}
$$

which is to be solved recursively for $k=1, \ldots, T / \tau$, starting with $y_{\tau}^{0}=y_{0}$. 
This boundary-value problem (in its suitable weak formulation) has a variational structure. More specifically, a weak solution can be obtained from the problem:

$$
\left.\begin{array}{ll}
\text { minimize } & \int_{\Omega} \varphi(\nabla y)+\mathscr{H}\left(\nabla^{2} y\right)+\tau \zeta\left(\nabla y_{\tau}^{k-1}, \frac{\nabla y-\nabla y_{\tau}^{k-1}}{\tau}\right)-f_{\tau}^{k} \cdot y \mathrm{~d} x \\
\text { subject to } & \text { meas } y(\Omega) \geq \int_{\Omega} \operatorname{det} \nabla y \mathrm{~d} x, \quad y=0 \text { on } \Gamma_{\mathrm{D}}, \quad y \in W^{2, p}\left(\Omega ; \mathbb{R}^{d}\right) .
\end{array}\right\}
$$

By the standard direct-method arguments, this problem has a solution which we will denote as $y_{\tau}^{k}$.

Comparing the value of the functional in the first line of (3.4) at $y=y_{\tau}^{k}$ with its value at $y=y_{\tau}^{k-1}$ which must be bigger of equal, and summing it for $k=1, \ldots, K$, we obtain the discrete energy imbalance for arbitrary $K \leq T / \tau$ :

$$
\begin{aligned}
& \int_{\Omega} \varphi\left(\nabla y_{\tau}^{K}\right)+\mathscr{H}\left(\nabla^{2} y_{\tau}^{K}\right) \mathrm{d} x+\tau \sum_{k=1}^{K} \int_{\Omega} \zeta\left(\nabla y_{\tau}^{k-1}, \nabla \frac{y_{\tau}^{k}-y_{\tau}^{k-1}}{\tau}\right) \mathrm{d} x \\
& \quad \leq \tau \sum_{k=1}^{K} \int_{\Omega} f_{\tau}^{k} \cdot \frac{y_{\tau}^{k}-y_{\tau}^{k-1}}{\tau} \mathrm{d} x+\int_{\Omega} \varphi\left(\nabla y_{0}\right)+\mathscr{H}\left(\nabla^{2} y_{0}\right) \mathrm{d} x . \\
& \quad=\int_{\Omega} f_{\tau}^{K} \cdot y_{\tau}^{K} \mathrm{~d} x-\tau \sum_{k=1}^{K} \int_{\Omega} y_{\tau}^{k-1} \cdot \frac{f_{\tau}^{k}-f_{\tau}^{k-1}}{\tau} \mathrm{d} x+\int_{\Omega} \varphi\left(\nabla y_{0}\right)+\mathscr{H}\left(\nabla^{2} y_{0}\right)-f_{\tau}^{0} \cdot y_{0} \mathrm{~d} x \\
& \quad \leq C\|f\|_{H^{1}\left(I ; L^{2}\left(\Omega ; \mathbb{R}^{n}\right)\right)} \sup _{0 \leq k \leq T / \tau}\left\|y_{\tau}^{k}\right\|_{L^{2}\left(\Omega ; \mathbb{R}^{n}\right)}+\int_{\Omega} \varphi\left(\nabla y_{0}\right)+\mathscr{H}\left(\nabla^{2} y_{0}\right)-f_{\tau}^{0} \cdot y_{0} \mathrm{~d} x .
\end{aligned}
$$

Considering $\left\{y_{\tau}^{k}\right\}_{k=0, \ldots, T / \tau}$, we introduce a notation for the piecewise-constant and the piecewise affine interpolants defined respectively by

$$
\begin{array}{llrl}
\bar{y}_{\tau}(t) & =y_{\tau}^{k}, \quad \underline{y}_{\tau}(t)=y_{\tau}^{k-1}, & & \text { and } \\
y_{\tau}(t) & =\frac{t-(k-1) \tau}{\tau} y_{\tau}^{k}+\frac{k \tau-t}{\tau} y_{\tau}^{k-1} & & \text { for }(k-1) \tau<t \leq k \tau .
\end{array}
$$

We will also use the notation $\bar{\sigma}_{\tau}$ and $\bar{f}_{\tau}$ with analogous meaning.

Since $\zeta \geq 0$, taking the supremum over $K$ in (3.5) and using the Poincaré inequality based on the Dirichlet condition and the coercivity (3.1b) and (3.1a) of $\mathscr{H}$ and $\varphi$, respectively, we obtain the a-priori estimate

$$
\left\|y_{\tau}\right\|_{L^{\infty}\left(I ; W^{2, p}\left(\Omega ; \mathbb{R}^{d}\right)\right)} \leq C,
$$

with some constant $C=C\left(I, f, y_{0}, \Omega, \Gamma_{\mathrm{D}}, p, s, d\right)>0$. Using [6], from (3.7a) and (3.1a) we can also deduce that $\operatorname{det} \nabla y_{\tau}>0$ and even that

$$
\left\|\frac{1}{\operatorname{det} \nabla y_{\tau}}\right\|_{L^{\infty}(Q)} \leq C
$$

In addition, by a variant of Korn's inequality [16] (cf. (3.8) below) and (3.7a), we can also exploit the coercivity (3.1e) of $\zeta$ in (3.5), which gives that

$$
\left\|\nabla \dot{y}_{\tau}\right\|_{L^{2}\left(Q ; \mathbb{R}^{d \times d}\right)} \leq C .
$$


More precisely, for the proof of (3.7c) we used the following generalized Korn inequality proved by W. Pompe [16], generalizing earlier results by P. Neff [13]:

$$
\left\|\nabla \dot{y}_{\tau}\right\|_{L^{2}\left(Q ; \mathbb{R}^{d \times d}\right)} \leq C\left\|F \nabla \dot{y}_{\tau}+\left(\nabla \dot{y}_{\tau}\right)^{\top} F^{\top}\right\|_{L^{2}\left(Q ; \mathbb{R}^{d \times d}\right)}
$$

for a field $F \in C\left(\bar{\Omega} ; \mathbb{R}^{d \times d}\right)$ with $\min _{\bar{\Omega}}$ det $F>0$, here applied in a further generalized form with $F:=\left(\nabla \bar{y}_{\tau}\right)^{\top}$, which is always contained in a fixed compact subset of the admissible fields $F$ due to the uniform bounds (3.7a) and (3.7b).

By the results from [14] applied to (3.4), we can claim that $y_{\tau}^{k} \in W^{2, p}\left(\Omega ; \mathbb{R}^{d}\right)$ satisfies also the identity

$$
\begin{aligned}
& \int_{\Omega} \mathbb{D}\left(\nabla y_{\tau}^{k-1}\right)\left(\left[\nabla \dot{y}_{\tau}^{k}\right]^{\top} \nabla\right.\left.y_{\tau}^{k-1}+\left[\nabla y_{\tau}^{k-1}\right]^{\top} \nabla \dot{y}_{\tau}^{k}\right):\left(\left[\nabla y_{\tau}^{k-1}\right]^{\top} \nabla z+[\nabla z]^{\top} \nabla y_{\tau}^{k-1}\right)+\varphi^{\prime}\left(\nabla y_{\tau}^{k}\right): \nabla z \\
&+\mathscr{H}^{\prime}\left(\nabla^{2} y_{\tau}^{k}\right) \vdots \nabla^{2} z \mathrm{~d} x=\int_{\Omega} f_{\tau}^{k} \cdot z \mathrm{~d} x+\int_{\Gamma_{\mathrm{N}}}\left(\nabla y_{\tau}^{k}\right)^{-\top} \vec{n} \cdot z \mathrm{~d} \sigma_{\tau}^{k}
\end{aligned}
$$

for all $z \in W^{2, p}\left(\Omega ; \mathbb{R}^{d}\right)$ with $\left.z\right|_{\Gamma_{\mathrm{D}}}=0$ and with some scalar-valued non-negative measure $\sigma_{\tau}^{k} \in \operatorname{Meas}^{+}\left(\Gamma_{\mathrm{N}}\right)$. Here, note that to apply [14], we have temporarily interpreted the $\zeta$-term as absorbed into the elastic energy density $\varphi$ with $\tau>0$ and $\nabla y_{\tau}^{k-1} \in C\left(\bar{\Omega} ; \mathbb{R}^{d \times d}\right)$ fixed; this possibly breaks frame indifference which is assumed but not exploited in [14. The expression $\mathfrak{s}_{\tau}^{k}:=\left(\left.\nabla y_{\tau}^{k}\right|_{\Gamma_{\mathrm{N}}}\right)^{-\top} \vec{n} \sigma_{\tau}^{k}$ occurring in the last integral was obtained in [14. It is in the position of a traction in direction of the outer normal in the actual deformed configuration which (up to a positive scalar factor) is given by $\left(\left.\nabla y_{\tau}^{k}\right|_{\Gamma_{\mathrm{N}}}\right)^{-\top} \vec{n}$. Also notice that both $\nabla y_{\tau}^{k}$ and its inverse $\left(\nabla y_{\tau}^{k}\right)^{-1}$ (by (3.7b) ) are uniformly bounded and (even Hölder) continuous on the closure of $\Omega$, and so are their traces on $\Gamma_{\mathrm{N}}$. In particular, the traction $\sigma_{\tau}^{k}$ itself is a measure.

As shown in [14], $\sigma_{\tau}^{k}$ therefore $\mathfrak{s}_{\tau}^{k}$ vanishes outside the self-contact set, i.e.,

$$
0=\sigma_{\tau}^{k}\left(\left\{x \in \Gamma_{N} \mid y_{\tau}^{k}(t, x) \neq y_{\tau}^{k}(\tilde{x}) \text { for all } \tilde{x} \in \Gamma_{N} \backslash\{x\}\right\}\right) .
$$

By comparison, we obtain an estimate on the measure $\left(\nabla y_{\tau}^{k}\right) \vec{n} \sigma_{\tau}^{k} \in \operatorname{Meas}\left(\Gamma_{\mathrm{N}} ; \mathbb{R}^{d}\right)$, but unfortunately in a bigger space than the space of measures. Namely, writing (3.9) in terms of the interpolants as

$$
\begin{aligned}
\int_{Q} \mathbb{D}\left(\nabla \underline{y}_{\tau}\right)\left(\left[\nabla \dot{y}_{\tau}\right]^{\top} \nabla \underline{y}_{\tau}\right. & \left.+\left[\nabla \underline{y}_{\tau}\right]^{\top} \nabla \dot{y}_{\tau}\right):\left(\left[\nabla \underline{y}_{\tau}\right]^{\top} \nabla z+[\nabla z]^{\top} \nabla \underline{y}_{\tau}\right)+\varphi^{\prime}\left(\nabla \bar{y}_{\tau}\right): \nabla z \\
& +\mathscr{H}^{\prime}\left(\nabla^{2} \bar{y}_{\tau}\right) \vdots \nabla^{2} z \mathrm{~d} x \mathrm{~d} t=\int_{Q} \bar{f}_{\tau} \cdot z \mathrm{~d} x \mathrm{~d} t+\int_{\Sigma_{\mathrm{N}}}\left(\nabla \bar{y}_{\tau}\right)^{-\top} \vec{n} \cdot z \mathrm{~d} \bar{\sigma}_{\tau}
\end{aligned}
$$

for all $z \in L^{1}\left(I ; W^{2, p}\left(\Omega ; \mathbb{R}^{d}\right)\right)$ with $\left.z\right|_{\Sigma_{\mathrm{D}}}=0$, we can estimate

$$
\begin{aligned}
& \sup _{\|z\|_{L^{2}\left(I ; W^{2-1 / p, p}\left(I_{\mathrm{N}} ; \mathbb{R}^{d}\right)\right)} \leq 1} \int_{\Sigma_{\mathrm{N}}}\left(\nabla \bar{y}_{\tau}\right)^{-\top} \vec{n} \cdot z \mathrm{~d} \bar{\sigma}_{\tau} \\
& \leq\left. C_{1} \sup _{\|z\|_{L^{2}\left(I ; W^{2, p}\left(\Omega ; \mathbb{R}^{d}\right)\right)} \leq 1} \int_{\Sigma_{\mathrm{N}}}\left(\nabla \bar{y}_{\tau}\right)^{-\top} \vec{n} \cdot z\right|_{\Sigma_{\mathrm{N}}} \mathrm{d} \bar{\sigma}_{\tau} \\
& =C_{1} \sup _{\|z\|_{L^{2}\left(I ; W^{2, p}\left(\Omega ; \mathbb{R}^{d}\right)\right)} \leq 1} \int_{Q} \mathbb{D}\left(\nabla \underline{y}_{\tau}\right)\left(\left[\nabla \dot{y}_{\tau}\right]^{\top} \nabla \underline{y}_{\tau}+\left[\nabla \underline{y}_{\tau}\right]^{\top} \nabla \dot{y}_{\tau}\right):\left(\left[\nabla \underline{y}_{\tau}\right]^{\top} \nabla z+[\nabla z]^{\top} \nabla \underline{y}_{\tau}\right) \\
& +\varphi^{\prime}\left(\nabla \bar{y}_{\tau}\right): \nabla z+\mathscr{H}^{\prime}\left(\nabla^{2} \bar{y}_{\tau}\right) \vdots \nabla^{2} z-\bar{f}_{\tau} \cdot z \mathrm{~d} x \mathrm{~d} t \\
& \leq C_{2}\left((\max |\mathbb{D}|)|| \nabla \underline{y}_{\tau}\left\|_{L^{\infty}\left(Q ; \mathbb{R}^{d \times d}\right)}\right\| \nabla \dot{y}_{\tau}\left\|_{L^{2}\left(Q ; \mathbb{R}^{d \times d}\right)}+\right\| \varphi^{\prime}\left(\nabla \bar{y}_{\tau}\right) \|_{L^{\infty}\left(Q ; \mathbb{R}^{d \times d}\right)}\right. \\
& \left.+\left\|\mathscr{H}^{\prime}\left(\nabla^{2} \bar{y}_{\tau}\right)\right\|_{L^{2}\left(I ; L^{p^{\prime}}\left(\Omega ; \mathbb{R}^{d \times d \times d}\right)\right)}+\|f\|_{L^{2}\left(I ; L^{2}\left(\Omega ; \mathbb{R}^{d}\right)\right)}\right)
\end{aligned}
$$


with some constants $C_{1}, C_{2}$ depending on $\Omega, d$ and $p$. Together with (3.7), this implies the estimate

$$
\left\|\left.\left(\nabla \bar{y}_{\tau}\right)^{-\top}\right|_{\Sigma_{\mathrm{N}}} \vec{n} \bar{\sigma}_{\tau}\right\|_{L^{2}\left(I ; W^{2-1 / p, p}\left(\Gamma_{\mathrm{N}} ; \mathbb{R}^{d}\right)^{*}\right)} \leq C .
$$

By the Poincaré inequality, ( $(3.7 \mathrm{c})$ together with the time-constant Dirichlet boundary conditions gives even the estimate on $\dot{y}_{\tau}$ in $L^{2}\left(I ; H^{1}\left(\Omega ; \mathbb{R}^{d}\right)\right)$.

The estimates (3.7 $\mathrm{a}, \mathrm{c})$ and $(3.13)$ hold for the piecewise constant interpolants $\bar{y}_{\tau}$ and $\underline{y}_{\tau}$, as well. Therefore, now we can select a subsequence converging for $\tau \rightarrow 0$ in the sense

$$
\begin{array}{ll}
y_{\tau} \rightarrow y, \quad \bar{y}_{\tau} \rightarrow y, \quad \text { and } \quad \underline{y}_{\tau} \rightarrow y & \text { weakly* in } L_{\mathrm{w}}^{\infty}\left(I ; W^{2, p}\left(\Omega ; \mathbb{R}^{d}\right)\right), \\
\dot{y}_{\tau} \rightarrow \dot{y} & \text { weakly in } L^{2}\left(I ; H^{1}\left(\Omega ; \mathbb{R}^{d}\right)\right), \\
\left(\left.\nabla \bar{y}_{\tau}\right|_{\Gamma_{\mathrm{N}}}\right) \vec{n} \bar{\sigma}_{\tau} \rightarrow \mathfrak{s}_{1} & \text { weakly in } L^{2}\left(I ; W^{2-1 / p, p}\left(\Gamma_{\mathrm{N}} ; \mathbb{R}^{d}\right)^{*}\right) .
\end{array}
$$

Note that the limit $y$ also inherits (3.7b) and the Ciarlet-Nečas condition $(2.12 \mathrm{~b})$ from $\bar{y}_{\tau}$, since for a.e. $t \in I, \bar{y}_{\tau} \rightarrow y$ in $C^{1}$ and meas $\bar{y}_{\tau}(\Omega) \rightarrow$ meas $y(\Omega)$ (see [12, Prop. 4.3], e.g.). Moreover, by the Aubin-Lions compact-embedding theorem (see [17, Lemma 7.7]) and its generalization for time derivative measures (see [17, Cor.7.9]), respectively, we also have that

$$
y_{\tau} \rightarrow y, \quad \bar{y}_{\tau} \rightarrow y, \quad \text { and } \quad \underline{y}_{\tau} \rightarrow y \quad \text { strongly in } L^{2}\left(I ; H^{1}\left(\Omega ; \mathbb{R}^{d}\right)\right) .
$$

We now want to pass to the limit in (3.11) as $\tau \rightarrow 0$. The only problematic term there is the one with $\mathscr{H}^{\prime}$, because the other terms converge strongly due to (3.14d) or are essentially linear (the dissipation term involving $\mathbb{D}$ is linear in $\nabla \dot{y}_{\tau}$, while its other factors converge strongly). We now exploit the strict monotonicity of $\mathscr{H}^{\prime}$ to obtain better convergence for $\nabla^{2} \bar{y}_{\tau}$. Consider the test functions

$$
z_{\tau}:=\phi \cdot\left(y-\bar{y}_{\tau}\right), \quad \text { where } \phi \in L^{\infty}\left(I ; C^{2}\left(\bar{\Omega} ; \mathbb{R}^{+}\right)\right) \text {such that } \int_{\Gamma} \phi \mathrm{d} \bar{\sigma}_{\tau}=0 \text { on } I \text { for all } \tau \text {. }
$$

Notice that $z_{\tau} \rightarrow 0$ strongly in $L^{2}\left(I ; H^{1}\left(\Omega ; \mathbb{R}^{d}\right)\right)$ and weakly in $L^{p}\left(I ; W^{2, p}\left(\Omega ; \mathbb{R}^{d}\right)\right)$ by (3.14d) and (3.14a). Using (3.11), we get that

$$
\begin{aligned}
& \limsup _{\tau \rightarrow 0} \int_{Q} \phi\left[\mathscr{H}^{\prime}\left(\nabla^{2} y\right)-\mathscr{H}^{\prime}\left(\nabla^{2} \bar{y}_{\tau}\right)\right]:\left[\nabla^{2}\left(y-\bar{y}_{\tau}\right)\right] \mathrm{d} x \mathrm{~d} t \\
& =\limsup _{\tau \rightarrow 0} \int_{Q}\left[\mathscr{H}^{\prime}\left(\nabla^{2} y\right)-\mathscr{H}^{\prime}\left(\nabla^{2} \bar{y}_{\tau}\right)\right] \vdots \nabla^{2} z_{\tau} \mathrm{d} x \mathrm{~d} t \\
& =\limsup _{\tau \rightarrow 0} \int_{Q}\left(-\mathbb{D}\left(\nabla \underline{y}_{\tau}\right)\left(\left[\nabla \dot{y}_{\tau}\right]^{\top} \nabla \underline{y}_{\tau}+\left[\nabla \underline{y}_{\tau}\right]^{\top} \nabla \dot{y}_{\tau}\right):\left(\left[\nabla \underline{y}_{\tau}\right]^{\top} \nabla z_{\tau}+\left[\nabla z_{\tau}\right]^{\top} \nabla \underline{y}_{\tau}\right)\right. \\
& \left.\quad-\varphi^{\prime}\left(\nabla \bar{y}_{\tau}\right): \nabla z_{\tau}+\int_{Q} \bar{f}_{\tau} \cdot z_{\tau}\right) \mathrm{d} x \mathrm{~d} t=0 .
\end{aligned}
$$

Since $\phi \in L^{\infty}\left(I ; C^{2}\left(\bar{\Omega} ; \mathbb{R}^{+}\right)\right)$was arbitrary apart from the requirement that $\int_{\Gamma} \phi \mathrm{d} \bar{\sigma}_{\tau}=0$ on $I$ for all (small enough) $\tau$, the strict monotonicity (3.1c) of $\mathscr{H}^{\prime}$ thus implies that

$$
\nabla^{2} y_{\tau} \rightarrow \nabla^{2} y \quad \text { in } L^{p}\left(I ; L^{p}\left(\Omega \backslash U_{t} ; \mathbb{R}^{d}\right)\right), \quad \text { i.e., } \int_{I} \int_{\Omega \backslash U_{t}}\left|\nabla^{2} y_{\tau}-\nabla^{2} y\right|^{p} \mathrm{~d} x \mathrm{~d} t \rightarrow 0
$$


for any measurable set $U \subset I \times \mathbb{R}^{d}, U=\bigcup_{t \in I}\{t\} \times U_{t}$, such that

$$
\begin{aligned}
& \text { the support of } \bar{\sigma}_{\tau}(t) \text { is contained in the interior of } U_{t} \\
& \text { for a.e. } t \in I \text { and (small enough) } \tau>0 .
\end{aligned}
$$

In particular, $y_{\tau} \rightarrow y$ strongly in $L^{p}\left(I ; W_{\text {loc }}^{2, p}\left(\Omega ; \mathbb{R}^{d}\right)\right)$ because $U:=I \times V$ is admissible for any closed neighborhood $V$ of $\Gamma$ in $\mathbb{R}^{d}$.

In view of (3.16) and (3.15), it is clear that for a.e. $t \in I$, the limit function $y$ inherits its trace on the boundary as a strong limit of the traces of $y_{\tau}$, except on the part $\Gamma_{t}^{*}$ of $\Gamma$ that is always excluded by $U_{t}$, that is,

$$
\Gamma_{t}^{*}:=\left\{x \in \Gamma_{\mathrm{N}} \mid \begin{array}{r}
x=\lim _{n \rightarrow \infty} x_{\tau(n)} \text { for a suitable subsequence }(\tau(n)) \text { of }(\tau) \\
\text { and points } x_{\tau(n)} \in \operatorname{supp} \bar{\sigma}_{\tau(n)}(t) \subset \Gamma_{\mathrm{N}}
\end{array}\right\} \subset \mathfrak{S}_{t} .
$$

Here, we indeed have that $\Gamma_{t}^{*} \subset \mathfrak{S}_{t}$ (the self-contact set of $y$ at time $t$, cf. Definition 2.1), due to (3.10), the definition of $\Gamma_{t}^{*}$ and the fact that $\bar{y}_{\tau}(t, \cdot) \rightarrow y(t, \cdot)$ strongly in $C\left(\bar{\Omega} ; \mathbb{R}^{d}\right)$, the latter by (3.14c) and compact embedding. Moreover, as a consequence of (3.10), (3.14c) and (3.16),

$$
\Gamma_{t}^{*} \text { contains the support of } \mathfrak{s}_{1}(t, \cdot) \in W^{2-1 / p, p}\left(\Gamma_{\mathrm{N}} ; \mathbb{R}^{d}\right)^{*} .
$$

As a consequence of $(3.15)$ and (3.1b),

$$
\mathscr{H}^{\prime}\left(\nabla^{2} \bar{y}_{\tau}\right) \rightarrow \mathscr{H}^{\prime}\left(\nabla^{2} y\right) \text { strongly in } L^{p^{\prime}}\left(I ; L^{p^{\prime}}\left(\Omega \backslash U_{t} ; \mathbb{R}^{d}\right)\right) .
$$

On the other hand, by (3.7a), passing to a subsequence if necessary, there exists $\mathfrak{h} \in L_{\mathrm{w}}^{\infty}\left(I ; W^{2, p}\left(\Omega ; \mathbb{R}^{d}\right)^{*}\right)$ such that

$$
\mathscr{H}^{\prime}\left(\nabla^{2} \bar{y}_{\tau}\right) \rightarrow \mathfrak{h} \text { weakly* in } L_{\mathrm{w}}^{\infty}\left(I ; W^{2, p}\left(\Omega ; \mathbb{R}^{d}\right)^{*}\right)=L^{1}\left(I ; W^{2, p}\left(\Omega ; \mathbb{R}^{d}\right)\right)^{*} .
$$

Interpreting $\mathscr{H}^{\prime}\left(\nabla^{2} y\right)$ as a distribution in $L^{1}\left(I ; W^{2, p}\left(\Omega ; \mathbb{R}^{d}\right)\right)^{*}$, i.e.,

$$
\left\langle\mathscr{H}^{\prime}\left(\nabla^{2} y\right), z\right\rangle:=\int_{Q} \mathscr{H}^{\prime}\left(\nabla^{2} y\right) \vdots \nabla^{2} z \mathrm{~d} x \mathrm{~d} t \quad \text { for } z \in L^{1}\left(I ; W^{2, p}\left(\Omega ; \mathbb{R}^{d}\right)\right),
$$

we see that

$$
\langle\mathfrak{h}, z\rangle=\left\langle\mathscr{H}^{\prime}\left(\nabla^{2} y\right), z\right\rangle \quad \forall z \in L^{1}\left(I ; W^{2, p}\left(\Omega ; \mathbb{R}^{d}\right)\right) \text { with } z=0 \text { on } \Sigma^{*}:=\bigcup_{t \in I}\left(\{t\} \times \Gamma_{t}^{*}\right) .
$$

In particular,

$$
\begin{aligned}
& \mathfrak{s}_{2}:=\mathfrak{h}-\mathscr{H}^{\prime}\left(\nabla^{2} y\right) \in L^{1}\left(I ; W^{2, p}\left(\Omega ; \mathbb{R}^{d}\right)\right)^{*} \quad \text { is supported in } \Sigma^{*}, \text { and } \\
& \mathfrak{s}_{2} \in L^{1}\left(I ; W^{2-\frac{1}{p}, p}\left(\Gamma_{N} ; \mathbb{R}^{d}\right)\right)^{*}=L_{\mathrm{w}}^{\infty}\left(I ; W^{2-\frac{1}{p}, p}\left(\Gamma_{N} ; \mathbb{R}^{d}\right)^{*}\right) .
\end{aligned}
$$

The latter holds because due to (3.21),$\left\langle\mathfrak{s}_{2}, z\right\rangle$ actually only depends on the traces of $z(t, \cdot)$ on $\Gamma_{t}^{*} \subset \Gamma_{N}, t \in I$. Altogether, we can now pass to the limit in (3.11), using (3.20), (3.14c) and (3.14d). This yields the limit equation (2.12a) with

$$
\mathfrak{s}:=\mathfrak{s}_{1}+\mathfrak{s}_{2} \in L^{2}\left(I ; W^{2-\frac{1}{p}, p}\left(\Gamma_{N} ; \mathbb{R}^{d}\right)^{*}\right) .
$$

Moreover, by (3.17), (3.18), and (3.22), the total contact reaction force $\mathfrak{s}$ satisfies (2.12c), and we conclude that $(y, \mathfrak{s})$ is a weak solution to the initial-boundary-value problem (2.8)-(2.9) $-(2.11)$ in the sense of Definition 2.1. 
Remark 3.2 (Open problem: actual reaction force) In the static situations, the contact reaction force $\mathfrak{s}$ is a measure as shown in [14, and it has a natural pullback to the reference configuration given by $\vec{n} \sigma=\left(\left.\nabla y\right|_{\Gamma_{\mathrm{N}}}\right)^{\top} \mathfrak{s}$. By contrast, in the evolution case, we are loosing this property, cf. the estimate (3.13), because $\left.\nabla y\right|_{\Sigma_{\mathrm{N}}}$ is not regular enough to justify multiplication with the distribution $\mathfrak{s}$. Additional information about the reaction force in the static case contained in the "complementary slackness principle" of [14] is also lost in the limit, as least if $\mathfrak{s}_{2}$ does not vanish.

Remark 3.3 (Open problem: dynamical problems) In many applications, inertia cannot be neglected. Yet, when inertial forces of the form $\varrho \ddot{y}$, with $\varrho>0$ a mass density in the reference configuration, would be involved in (2.8), serious difficulties would occur in (3.12) where now

only the sum $\int_{\Sigma_{\mathrm{N}}}\left(\nabla \bar{y}_{\tau}\right)^{-\top} \vec{n} \cdot z \mathrm{~d} \bar{\sigma}_{\tau}+\int_{Q} \varrho \ddot{y}_{\tau} \cdot z \mathrm{~d} x \mathrm{~d} t$ could be estimated. As a result, in the limit problem, one could not distinguish between inertial forces and reaction forces arising from the possible self-contact. Thus, one would have to devise a very weak solution concept.

\section{Acknowledgements}

This research has been supported by the Czech Science Foundation through the grants 17-04301S (in particular concerning dissipative evolutionary systems), 19-29646L (especially pertaining large strains in materials science) and 19-04956S (in particular concerning nonlinear behavior of structures). Also the institutional support RVO:61388998 is acknowledged.

\section{References}

[1] S.S. Antman. Physically unacceptable viscous stresses. Zeitschrift angew. Math. Physik, 49:980-988, 1998.

[2] S.S. Antman. Nonlinear Problems of Elasticity. Springer, New York, 2nd edition, 2005.

[3] R.C. Batra. Thermodynamics of non-simple elastic materials. J. Elasticity, 6:451-456, 1976.

[4] P.G. Ciarlet and J. Nečas. Injectivity and self-contact in nonlinear elasticity. Arch. Ration. Mech. Anal., 97:171-188, 1987.

[5] E. Fried and M.E. Gurtin. Tractions, balances, and boundary conditions for nonsimple materials with application to liquid flow at small-lenght scales. Arch. Ration. Mech. Anal., 182:513-554, 2006.

[6] T.J. Healey and S. Krömer. Injective weak solutions in second-gradient nonlinear elasticity. ESAIM: Control, Optim. \& Cal. Var., 15:863-871, 2009.

[7] M. Kružík and T. Roubíček. Mathematical Methods in Continuum Mechanics of Solids. Springer, Switzerland, 2019.

[8] A. Mielke, C. Ortner, and Y. Sengül. An approach to nonlinear viscoelasticity via metric gradient flows. SIAM J. Math. Anal, 46:1317-1347, 2013.

[9] A. Mielke, R. Rossi, and G. Savaré. Global existence results for viscoplasticity at finite strain. Arch. Ration. Mech. Anal., 227, 2018. 
[10] A. Mielke and T. Roubíček. Rate-Independent Systems - Theory and Application. Springer, New York, 2015.

[11] A. Mielke and T. Roubíček. Thermoviscoelasticity in Kelvin-Voigt rheology at large strains. Archive Ration. Mech. Anal., Submitted. (Preprint: arXiv 1903.11094).

[12] A. Mielke and T. Roubíček. Rate-independent elastoplasticity at finite strains and its numerical approximation. Math. Models Methods Appl. Sci., 26:2203-2236, 2016.

[13] P. Neff. On Korn's first inequality with non-constant coefficients. Proc. Royal Soc. Edinburgh, 132A:221-243, 2002.

[14] A.Z. Palmer and T.J. Healey. Injectivity and self-contact in second-gradient nonlinear elasticity. Calc. Var., 56:Art. no. 114, 2017.

[15] P. Podio-Guidugli. Contact interactions, stress, and material symmetry, for nonsimple elastic materials. Theor. Appl. Mech., 28-29:261-276, 2002.

[16] W. Pompe. Korn's First Inequality with variable coefficients and its generalization. Comment. Math. Univ. Carolinae, 44:57-70, 2003.

[17] T. Roubíček. Nonlinear Partial Differential Equations with Applications. Birkhäuser, Basel, 2nd edition, 2013.

[18] F. Schuricht. Variational approach to contact problems in nonlinear elasticity. Calc. Var., 15:433-449, 2002.

[19] M. Šilhavý. Phase transitions in non-simple bodies. Arch. Ration. Mech. Anal., 88:135-161, 1985.

[20] R.A. Toupin. Elastic materials with couple stresses. Arch. Ration. Mech. Anal., 11:385-414, 1962.

[21] R.A. Toupin. Theories of elasticity with couple stress. Archive Ration. Mech. Anal., 17:85$112,1964$.

[22] N. Triantafyllidis and E.C. Aifantis. A gradient approach to localization of deformation. I. Hyperelastic materials. J. Elast., 16:225-237, 1986.

[23] B. Tvedt. Quasilinear equations of viscoelasticity of strain-rate type. Arch. Ration. Mech. Anal., 189:237-281, 2008. 